\title{
The pyruvate kinase activity in peripheral and uterine blood in women with atypical endometrial hyperplasia and endometrial cancer
}

\section{Type}

Research paper

\section{Keywords}

Endometrial Cancer, atypical endometrial hyperplasia, aerobic glycolysis, PKM2

\begin{abstract}
Introduction

Pyruvate kinase in an enzyme that catalyzes the production of pyruvate and ATP as the final step of the glycolysis. Potential role of Pyruvate kinase in tumorigenesis was previously suggested, due to its altered activity in several tumors.

\section{Material and methods}

This study looks at M2 isozyme of pyruvate kinase activity (M2-PK) measured in peripheral and uterine blood plasma in various stages of endometrial cancer $(\mathrm{EC})$ as well as in precancerous state of atypical endometrial hyperplasia (AEH). Measurements were performed using spectrophotometric method in citrate plasma samples from peripheral and uterine blood. Study group included 84 patients with endometrial cancer, 28 patients with atypical endometrial hyperplasia and 23 noncancerous controls.
\end{abstract}

\section{Results}

Pyruvate kinase activity in EC group was 3-fold higher than in control group both in peripheral and uterine blood samples. Pyruvate kinase activity was also 3-fold higher in uterine blood when compared to peripheral blood samples. We also found statistically significant correlation between FIGO staging and detected activity with the M2-PK activity being 2-fold higher for FIGO1 than for FIGO3. We also describe a paradox in which the M2-PK activity in patients with atypical endometrial hyperplasia is lower than M2-PK activity in control group in peripheral blood samples, but higher in uterine blood samples.

\section{Conclusions}

The measurement of citrate plasma pyruvate kinase metabolic activity varies greatly between samples collected from different sites and samples collected from patients with varied tumor staging. Further studies are needed in order to elucidate molecular pathways that are responsible for observed differences. 


\section{Introduction}

Endometrial cancer (EC) is one of the most common gynecological malignancies, affecting more than 287,000 women annually and contributing to 74,000 deaths worldwide EC develops from the uterine mucosa (endometrium), most often in post-menopausal women [1]. The main methods of treatment include total laparoscopic or total abdominal hysterectomy [2]. While EC has been wellstudied at the genetic level, the metabolic basis of this disease has not yet been fully described. It is widely known that obesity is one of the greatest cancer risk factors and it has been shown that not only body mass index $(\mathrm{BMI})>30 \mathrm{~kg} / \mathrm{m} 2$ but also hyperglycemia in type 1 and 2 diabetes are associated with an increased incidence, risk of death, and lower age of EC diagnosis. This means that any disturbances in the glucose metabolism may play an important role in in the development and growth of EC.

The knowledge that tumor cells demonstrate altered metabolic phenotype has been with us since 1920. The alterations characterised by an elevated glucose uptake rate and reduced rates of oxidative phosphorylation. This process of high lactate production by tumor cells in the presence of oxygen was described by Otto Warburg [3]. Warburg demonstrated that tumor cells use aerobic glycolysis with reduced mitochondrial oxidative phosphorylation for glucose metabolism and that the aerobic glycolysis derives from mitochondrial injury. He hypothesized that this mechanism is an early, essential, and irreversible step in a tumorigenesis. Recent studies have demonstrated however, that tumor cells can turn back to mitochondrial respiration under specific circumstances. It has been suggested that there must be an association between aerobic glycolysis in tumor cells and oncogene activation or tumor suppressor gene suppression.

Pyruvate kinase $(P K)$ is an enzyme that catalyzes the transfer of a phosphate group from phosphoenolpyruvate (PEP) to adenosine diphosphate (ADP), a process which produces pyruvate and ATP as the final step of glycolysis [4]. The potential role of PK in tumorigenesis has been suggested by several studies. It has been shown that PKM2, being the embryonic isoform of enzyme with its transcription attenuated in some adult tissues, is reactivated in several tumors such as EC [5]. What is more, a switch from the PKM1 to the PKM2 isoform has been observed in various cancers such as hepatocellular carcinoma [6] with c-Myc oncogene affecting M-gene splicing in a way that favors PKM2 [7]. Several connections regarding modified PKM2 activity affecting tumor growth in vivo were also observed [8,9]. In addition, it has been proposed that PKM2 is involved in intracellular signaling modulator by its ability to interact with several proteins such as tyrosine kinases (BCR-ABL, A Raf, FGFR1) which are able to modulate the dimeric/tetrameric state of PKM2 thus affecting cell metabolism [10]. Several links between PKM2 and the anti-apoptotic Bcl2 protein have also been observed [11]. These results imply that PKM2 may possess translation-controlling properties while also helping cancer cells with oxidative stress and extracellular insult adaptation through intra-mitochondrial Bcl2 expression protection. PK has also been shown to enhance tumor angiogenesis by acting as a transcriptional factor interacting with HiF-1a and activating its target gene VEGF-A - which increases blood vessel formation thus contributing to tumor growth [12]. PKM2 can also be transported as a package protein of exosomes acting as an extracellular signaling molecule. Several studies have indicated that circulating PKM2 
may be responsible for tumor growth through angiogenesis, migration and matrix adhesion of endothelial cells [13]. Even though some studies do not support the oncogenic role of PKM2 (with PKM2 knockout mice having an accelerated BRCA1 driven tumor formation) its overexpression has been universally connected to several human cancers as well as poor clinical outcome [14]. These cancers include gastric cancer, hepatocellular carcinoma, breast cancer and ovarian cancer. Due to this low specificity it is unlikely to serve as an useful screening marker on its own, but could serve as such when combined with other markers by increasing thier sensitivity and specificity [15].

Endometrial hyperplasia in patients with PCOS has been associated with PKM2 overexpression when compared to patients without PCOS or without hyperplasia [16]. It also indicates that PKM2-driven glycolysis inhibition with increased mitochondrial activity may contribute to the onset of ER-dependent endometrial hyperplasia. Several attempts to assess if metabolic switches precede or follow the histological changes have also been undertaken. One of them has shown lowered PKM1 expression in patients with EC compared to non-cancerous patients implicating that disappearance of PKM1 may be associated with cancer phenotype progression and could serve as a prognostic factor in patients with endometrial hyperplasia [3]. Some studies have explored the idea of PK serving as an EC marker, with promising results [17,18].

In this study we explored whether these previously mentioned metabolic alterations precede histological changes in precancerous lesions. We wanted to evaluate whether altered pyruvate kinase M2 activity is detectable in the precancerous state of atypical endometrial hyperplasia as well as to investigate whether PKM2 activity differs across different stages of endometrial cancer. There were 3 objectives to this study. The first was to determine whether altered pyruvate kinase M2 in plasma in comparison to non-neoplastic controls can be detected not only in endometrial cancer but also in the precancerous state of atypical endometrial hyperplasia. The second goal was to determine whether pyruvate kinase M2 activity differs between peripheral and uterine blood of patients with $\mathrm{AEH}$ and endometrial cancer. The third goal was to determine whether plasma pyruvate kinase M2 activity varies between the different stages of EC according to the FIGO classification.

\section{Materials and Methods}

The study was approved by the Jagiellonian University Bioethics Committee (approval number 1072.6120.96.2017). All subjects included in the study gave their written informed consent. The endometrial cancer group was made up of 84 patients aged $40-75$. Their medical histories were taken and a record was made of who had histologically proven endometrial cancer. 28 women with atypical endometrial hyperplasia $(\mathrm{AEH})$ were included in the second group. The control group consisted of 23 women aged $40-65$ who had undergone hysterectomies due to non-cancerous reasons.

Peripheral and uterine venous blood samples were collected into tubes containing citrate as anticoagulant. Both peripheral and uterine blood were collected from the peripheral vein and uterine vein, respectively. The patients were operated on by laparotomy or laparoscopy. After an inspection of the pelvis and the abdominal cavity, blood was collected from the venous vessels of the so-called 
uterine venous plexus, which is located between the plaques of the broad ligament along the lateral circumference of the uterine body. Blood sample collection was performed during surgical procedures at the Department of Gynecological Oncology, Jagiellonian University Medical College. The procedure was as follows: We chose citrate plasma samples due to the proven high stability and ability to acurately reproduce results. Samples were then allowed to stand at room temperature for 30 minutes after which they were centrifuged at $14000 \mathrm{rpm}$ for 10 minutes and the plasma was stored at $-80^{\circ} \mathrm{C}$.

Total pyruvate kinase activity M2 was determined using the Pyruvate Colorimetric/Fluorometric assay kit (BioVision; Milpitas, CA, USA). The assay procedure was performed according to manufacturer's instructions. Pyruvate concentration was measured spectrophotometrically at 570 $\mathrm{nm}$ in $10 \mathrm{ul}$ of plasma. Optical density was measured at $570 \mathrm{~nm}$ at two-minutes intervals for 15 minutes using a SpectraMax Gemini EM multiplate fluorescence reader. The mean signal intensity was averaged across 3 biological replicates. Pyruvate kinase activity was then calculated based on two absorbance readings in the linear reaction range. Results were expressed in $\mathrm{mU} / \mathrm{mL}$. We measured pyruvate kinase activity in citrate plasma samples from peripheral and uterine blood using Pyruvate Kinase Activity Colorimetric assay kit (BioVision). In that assay, phosphoenolpyruvate (PEP) and ADP are catalyzed by pyruvate kinase to generate pyruvate and ATP. The generated pyruvate is oxidized by pyruvate oxidase in order to produce color (at $\lambda=570$ $\mathrm{nm}$ ) and fluorescence (at $E x / E m=535 / 587 \mathrm{~nm}$ ). The pyruvate kinase activity is detected when the increase in colour or fluorescence intensity is proportional to the increase in amount of pyruvate. The detection threshold for this method is $0.1 \mathrm{mU} / \mathrm{ml}$.

\subsection{Statistical analyses}

The statistical analyses were performed using SPSS statistics 20.0 software (IBM, Armonk, NY, USA). T-test was applied to evaluate the differences of pyruvate kinase activity between healthy controls and patients with atypia or endometrial cancer as well as between peripheral and uterine blood samples. The activity of pyruvate kinase in plasma of patients with different endometrial cancer stages (according to FIGO) was compared with atypical hyperplasia of endometrium. Statistical tests were one/two sided and $p<0.05$ was considered statistically significant. Non-parametric Kruskal-Wallis one-way ANOVA based on ranks was employed to assess differences between median values. The differences between more than two the mean values were assessed based on analysis of variance (ANOVA). To show for which pair of comparisons the difference was significant, Tukey's post hock test was used.

\section{Results}

3.1. Pyruvate kinase M2 activity in the plasma samples from peripheral blood of patients with atypia or endometrial cancer in comparison to healthy controls

We found statistically significant differences in mean M2-PK levels between all three study groups:. It can be seen from Figure 1 that the PK activity was the lowest $(<9.5 \mathrm{mU} / \mathrm{mL})$ in group of patients 
with atypical endometrial hyperplasia.. We observed a rise (from about 9 to above $12 \mathrm{mU} / \mathrm{mL}$ ) of pyruvate kinase activity in patients with endometrial cancer in relation to patients with AEC. The pyruvate kinase values in patients with endometrial cancer ranged from above 11.5 to $12.5 \mathrm{mU} / \mathrm{mL}$ with a mean value $12.0 \mathrm{mU} / \mathrm{mL}$. The post-hock tests analysis showed statistical significances in pairs between all groups $(p<0.01)$.

The analysis of variance (ANOVA) between the same samples with EC group divided by the FIGO staging can be seen in Figure 2. The post-hock tests were performed to show differences between the samples in pairs. We found statistically significant differences $(p=2.18 e-12)$ in mean values of pyruvate kinase activity in the peripheral blood plasma samples of women with endometrial cancer striated into FIGO IA, FIGO IB, FIGO>III groups. The highest statistical significances were seen between all FIGO stages and atypia $(p<0.00)$, as well as control - atypia $(p<0,03)$ and FIGO IA control $(p<0.00)$, FIGO $>$ III - control $(p<0.00)$.

\subsection{Pyruvate kinase M2 activity in citrate plasma samples from uterine blood of patients with atypia} or endometrial cancer in comparison to healthy controls

A comparison of the pyruvate kinase activity in uterine blood of women with $\mathrm{AEH}$ or women with endometrial cancer and healthy controls can be seen in Figure 3. We found statistically significant differences in the mean pyruvate kinase levels between the control group having a mean value of $26 \mathrm{mU} / \mathrm{mL}$, atypia having a mean value of $29 \mathrm{mU} / \mathrm{mL}$ and endometrial cancer group having a mean value of $47,5 \mathrm{mU} / \mathrm{mL}$. In uterine blood pyruvate kinase activity was more than three times as high as in samples from peripheral blood $(p=7.5 e-12)$. The post-hock test showed differences between the samples in pairs. The mean pyruvate kinase activity was significantly different in endometrial cancer samples: endometrial cancer vs control $(p<0.000)$ and endometrial cancer vs atypia $(p<0,005)$.

An analysis of variance (ANOVA) including FIGO classification for endometrial cancer was also performed, results are presented on Figure 4. Pyruvate kinase activity in uterine blood of patients with endometrial cancer ranged from 30 to $60 \mathrm{mU} / \mathrm{mL}$ and its mean values differed significantly between cancer stages according to FIGO scale, with a lower detected activity for more advanced staging. The statistically significant differences were found in following pairs:

FIGO IA - AEH $(p<0.001)$

FIGO IB - AEH $(p<0.004)$,

FIGO IA - control $(p<0.001)$

FIGO IB - control $(p<0.001)$.

FIGO $>$ III was statistically different only in comparison to FIGO IA $(p<0.001)$ and FIGO IB $(p<0.005)$.

3.3. Pyruvate kinase $M 2$ activity in citrate plasma samples from peripheral and uterine blood of patients with different endometrial cancer stages according to FIGO classification and in atypia 
Figures 5 and 6 demonstrate differences in pyruvate kinase activity between peripheral and uterine blood samples. The most prominent findings include pyruvate kinase activity in uterine blood being almost three times higher than in peripheral blood for atypia samples $(p=2.2 e-16$, Figure 5$)$, and almost twice as high for EC samples ( $p=2.341 \mathrm{e}-15$, Figure 6$)$. In atypia samples from uterine blood the mean value was about 3 times as high when compared to peripheral blood samples. This tendency was significant for all stages of the disease (FIGO IA, FIGO IB, FIGO >III, $p<0.0001$ ).

\section{Discussion}

Endometrial cancer is the second most common and the fourth leading cause of death due to gynecological cancer among women worldwide [19]. The incidence rate of that tumor is increasing rapidly, and is estimated to increase by more than $50 \%$ worldwide by 2040 [20]. About $40 \%$ of EC cases are still diagnosed at advanced stages with early metastasis and a poor survival rate. Hyperplasia of endometrium is very significant clinically due to the associated risk of progression to endometrioid EC. Nowadays, atypical forms of EH are recognized as premalignant lesions. Differential diagnosis between $\mathrm{AH}$ and $\mathrm{EC}$ is highly essential for cytological atypia. In this study we concentrated on pyruvate kinase activity in patients with EC at different clinical stages (based on FIGO classification) and with AEH.

The results showed that pyruvate kinase M2 activity differs across different clinical stages of the disease, indicating its potential role in carcinogenesis. We demonstrated that peripheral blood plasma pyruvate kinase M2 activity was lowest in patients with $\mathrm{AEH}$, with the highest activity being observed for endometrial cancer group. During tumorigenesis, the reprogramming of cell metabolism is critical. Cancer cells are characterized by a high glycolytic rate, which leads to energy regeneration and anabolic metabolism. It is known that tumor cells are primarily dependent on aerobic glycolysis to obtain energy and produce lactate, even in the presence of oxygen. It was suggested in many studies that PKM2 is involved in a complex network that regulates cancer cell metabolism. The studies showed that PKM2 was upregulated in proliferating cells such as cancerous and embryonic cells [21]. It was demonstrated that proliferating cells are able to reprogram their cellular glucose metabolism based on their bioenergetics and biosynthetic needs in order to maintain a proper cellular redox homeostasis. PKM2, as a critical regulator of this metabolic reprogramming, may be involved in cancer progression, regulating both metabolic and nonmetabolic pathways [21]. Exploring the role of PKM2 is essential because of the therapeutic relevance of anticancer drugs that could inhibit PKM2 activity. It has previously been shown that the downregulation of PKM2 expression, using inhibitors or short interfering RNA was possible in bladder cancer [22]. In the study of Salama A. et al., conducted on human endometrial cells, it was demonstrated that estradiol-17 $\beta$ (E2) treatment induced Warburg-like glucose metabolism by inducing the expression of PKM. Moreover, the authors showed that E2 enhanced PKM splicing into PKM2 as well as inducing PKM2 oxidation, phosphorylation, and nuclear translocation [24]. In that study it was demonstrated that PKM2 was able to interact with estrogen receptor- $\alpha(E R \alpha)$ and 
thus to play a role as an ERa coactivator. It was suggested that small-molecule PKM2 activators ameliorated ERa transcriptional activity and abrogated the E2-induced human primary endometrial stromal cells proliferation [23].

The deviation from the Warburg effect may be explained by the fact that tyrosine phosphorylation of M2-PK disrupts FBP binding, inhibiting the formation of the highly active tetramer conformation and lowering enzymatic activity. Phosphorylation promotes increased glucose consumption followed by increased lactate production and an exponential increase in cell proliferation. Factors such as post-menopausal age, obesity and elevated estrogen levels contribute to the post-translational modifications of M2-PK and provoke a decrease in activity, which can lead to higher level of tumor proliferation [24]. This theory correlates precisely with the observations from our study. A decrease in M2-PK activity leads to an increase in the production of glycolytic intermediates for biosynthetic pathways such as pentose phosphate pathway and serine biosynthesis pathways. Decreased pyruvate kinase activity has also been described as a factor provoking the increase in an alternative glycolytic pathway due to the decrease in M2-PK activity increasing PEP levels. PEP is a M2-PK substrate and is able to promote tumorigenesis. The increase in the level of PEP was also previously observed to cause the phosphorylation of phosphoglycerate mutase 1 (PGAM1) at histidine 11 position leading to the activation of PGAM1 which results in an increase of glycolysis and production of glycolytic intermediates [25]. M2-PK is one of the glycolytic targets of tyrosine kinase signaling and is thought to be one of the rates limiting the steps of glycolysis, playing an important role in the regulation of glycolytic activity. The posttranslational modifications of $\mathrm{M} 2-\mathrm{PK}$, such as phosphorylation, promote an increased glycolytic rate resulting in tumor cell proliferation. In that case this enzyme plays a pivotal role in enhancing the Warburg effect by increasing glycolysis and lactate production during tumorigenesis [26].

The novel part of this study is the comparison of PK activity in both uterine and peripheral blood samples. When looking at uterine blood samples, we can see that the detected levels of PK activity were higher in atypical endometrial hyperplasia group than in control group samples. We speculate that the reason that this increased activity was not detectable in peripheral blood samples was due to the fact that the markers of early stage of tumorigenesis may only have been present in close proximity to the tumor. It can be presumed with high probability that similar relationships may occur in the blood that comes out of the uterus during abnormal bleeding and may be used for testing in a non-invasive manner. It is generally known that abnormal uterine bleeding occurs in the early stages of the development of atypical endometrial hyperplasia and endometrial cancer.

last part of our study looked at the correlations between pyruvate kinase activity and tumor staging based on FIGO classification which reflects the clinical stage of the cancer. We detected that the pyruvate kinase activity was highest for early stage cancers (FIGO1A) and lower in more advanced cancers. These findings are in contrast to previous reports, which show an increase in plasma M2-PK levels with increasing tumor stages for various cancers such as gastric, pancreatic and lung cancer $[27,28]$. However, we suggest that the observed higher activity of pyruvate kinase in FIGO $1 \mathrm{~A}$ and lower in FIGO III stages may be related to the cell proliferation. Warburg-like 
glucose metabolism is characterized by increased glucose uptake and shifting the glucose metabolism toward aerobic glycolysis. This metabolic phenotype, strongly related to cell growth and proliferation, generates the energy, the anabolic precursors, and the redox equivalents required for the de novo synthesis of macromolecules and to remove ROS produced during cell proliferation [29-31]. The metabolic reprogramming is induced by signal pathways driving cell proliferation [32]. FIGO III is a stage of EC with advanced clinical symptoms and bigger tumors when compared with FIGO I and II, however the cell proliferation in this stage is lower than in FIGO I and II. It could explain the observed lower activity of pyruvate kinase in patients with the most clinically advanced EC.

Our study has several limitations. Due to low number of samples from patients with FIGO II and FIGO IV staging, we had to divide patients into just three groups to take statistical measurements: FIGO1A, FIGO1B and FIGO>3. Such action reduces the credibility of data regarding usage of $\mathrm{PK}$ activity measurement as a novel staging tool alternative to histopathological specimen analysis. More detailed studies regarding M2-PK activity in endometrial cancer are therefore needed in order to answer the question whether there is a link between M2-PK activity and endometrial cancer stages. Moreover, the molecular mechanism behind the paradox of the disparity of the pyruvate kinase activity that we observed in peripheral and uterine blood plasma of patients with atypia and endometrial cancer is still not clear. Lower M2-PK activity in FIGO 3 group when compared to FIGO $1 \mathrm{~A}$ group is also a subject for further studies dealing with the molecular basis of this relationship. According to numerous studies, including ours, M2-PK plays an important role in tumor metabolism and, therefore, it may be a potential goal of targeted therapy. Researches on substances modulating the activity of M2-PK are ongoing [33-35].

\section{Conclusions}

To conclude, our study demonstrates decreased pyruvate kinase activity in the pre-cancerous state of endometrium which is in line with the results of previous studies. This low pyruvate kinase activity result of the accelerated tumor growth. We also demonstrated that this activity is higher in samples collected in close proximity to atypia. We found significant differences in uterine pyruvate kinase activity compared to peripheral blood samples. EC is associated with uterine bleeding and in future studies, this material (excluding perioperative uterine blood samples) may be considered appropriate for testing for pyruvate kinase activity. We believe that this study can provide a direction for further studies assessing the involvement of pyruvate kinase activity in transition from a precancerous to cancerous state in endometrial cancers.

\section{Acknowledgments}

This work was reviewed by McGregor Language School.

\section{References}


1. Colombo N, Creutzberg C, Amant F, et al. ESMO-ESGO-ESTRO Consensus Conference on Endometrial Cancer: diagnosis, treatment and follow-up [published correction appears in Ann Oncol. $2017 \quad$ Jul 1;28(suppl_4):iv167-iv168]. Ann Oncol. 2016;27(1):16-41. doi:10.1093/annonc/mdv484

2. Tupacz-Mosakowska E, Abacjew-Chmyłko A, Wydra D. Laparoscopy and laparotomy in endometrial cancer - a meta-analysis. Archives of Medical Science. 2021.

3. Hosseini NS, Jooya N, Esmaeili A, Zarrin KN, Diaz-Arrastia C, Momeni M. Using Pyruvate Kinase as a Predictor for Patient with Endometrial Cancer Having Complex Hyperplasia With Atypia to Prevent Hysterectomy and Preserve Fertility: Retrospective Immunohistochemical Study. Reproductive Sciences 2017;25(8), 1286-1291. doi: 10.1177/1933719117741371

4. Gupta V, Bamezai RN. Human pyruvate kinase M2: a multifunctional protein. Protein Science 201019 (11): 2031-44. doi: 10.1002/pro.505

5. Christofk HR, Vander Heiden MG, Harris MH, et al. The M2 splice isoform of pyruvate kinase is important for cancer metabolism and tumour growth. Nature. 2008;452:230-3. doi: 10.1038/nature06734

6. Iqbal MA, Gupta V, Gopinath P, Mazurek S, Bamezai RN. Pyruvate kinase M2 and cancer: an updated assessment. FEBS Lett. 2014;588:2685-92. doi: 10.1016/j.febslet.2014.04.011

7. David CJ, Chen M, Assanah M, Canoll P, Manley JL. HnRNP proteins controlled by c-Myc deregulate pyruvate kinase mRNA splicing in cancer. Nature. 2010;463:364-8. doi: 10.1038/nature08697

8. Spoden GA, Mazurek S, Morandell D, et al. Isotype-specific inhibitors of the glycolytic key regulator pyruvate kinase subtype M2 moderately decelerate tumor cell proliferation. Int $\mathrm{J}$ Cancer. 2008;123:312-21. doi: 10.1002/ijc.23512

9. Li H, Lin X, Li C, et al. MiR-1286 inhibits lung cancer growth through aerobic glycolysis by targeting PKM2. Archives of Medical Science. 2019. doi:10.5114/aoms.2019.87812.

10. Hitosugi $T$, Kang $S$, Vander Heiden MG, et al. Tyrosine phosphorylation inhibits PKM2 to promote the Warburg effect and tumor growth. Sci Signal. 2009;2:ra73. doi: 10.1126/scisignal.2000431

11. Liang J, Cao R, Wang $X$, et al. Mitochondrial PKM2 regulates oxidative stress-induced apoptosis by stabilizing Bcl2. Cell Res. 2017;27:329-51. doi: 10.1038/cr.2016.159

12. Azoitei N, Becher A, Steinestel K, et al. PKM2 promotes tumor angiogenesis by regulating HIF1alpha through NF-kappaB activation. Mol Cancer. 2016;15:3. doi: 10.1186/s12943-015-04902

13. Li L, Zhang Y, Qiao J, Yang JJ, Liu ZR. Pyruvate kinase M2 in blood circulation facilitates tumor growth by promoting angiogenesis. J Biol Chem. 2014;289:25812-21. doi: 10.1074/jbc.M114.576934

14. Hsu MC, Hung WC. Pyruvate kinase M2 fuels multiple aspects of cancer cells: from cellular metabolism, transcriptional regulation to extracellular signaling. Mol Cancer 2018 Feb 19;17(1):35. doi: 10.1186/s12943-018-0791-3

15. Fung KY, Tabor B, Buckley MJ, et al. Blood-based protein biomarker panel for the detection of colorectal cancer. PLoS One. 2015;10:e0120425. doi: 10.1371/journal.pone.0120425 
16. Wang $\mathrm{T}$, Zhang J, Hu M, et al. Differential Expression Patterns of Glycolytic Enzymes and Mitochondria-Dependent Apoptosis in PCOS Patients with Endometrial Hyperplasia, an Early Hallmark of Endometrial Cancer, In Vivo and the Impact of Metformin In Vitro. Int J Biol Sci 2019; 15(3):714-725.. doi: 10.7150/ijbs.31425

17. DeSouza LV, Romaschin AD, Colgan TJ, Siu KWM. Absolute quantification of potential cancer markers in clinical tissue homogenates using multiple reaction monitoring on a hybrid triple quadrupole/linear ion trap tandem mass spectrometer. Anal Chem 200981 (9), 3462-3470. doi: $10.1021 / a c 802726 a$

18. Galazis N, Pang YL, Galazi M, Haoula Z, Layfield R, Atiomo W. Proteomic biomarkers of endometrial cancer risk in women with polycystic ovary syndrome: a systematic review and biomarker database integration, Gynecological Endocrinology, 2013 29:7, 638-644. doi: 10.3109/09513590.2013.777416

19. Bray F, Ferlay J, Soerjomataram I, Siegel RL, Torre LA, Jemal A. Global cancer statistics 2018: GLOBOCAN estimates of incidence and mortality worldwide for 36 cancers in 185 countries. CA Cancer J Clin. (2018) 68:394-424

20. Morice P, Leary A, Creutzberg C, Abu-Rustum N, Darai E. Endometrial cancer. Lancet. (2016) 387:1094-108

21. Hsu MC and Hung WC: Pyruvate kinase M2 fuels multiple aspects of cancer cells: From cellular metabolism, transcriptional regulation to extracellular signaling. Mol Cancer. 17:352018.

22. Chen J, Xie J, Jiang Z, Wang B, Wang $Y$ and Hu X: Shikonin and its analogs inhibit cancer cell glycolysis by targeting tumor pyruvate kinase-M2. Oncogene. 30:4297-4306. 2011.

23. Salama A. Salama, Mahmoud A. Mohammad, Concepcion R. Diaz-Arrastia,Marwa W. Kamel, Gokhan S. Kilic, Bih T. Ndofor, Mohamed S. Abdel-Baki, andShaleen K. Theiler, Estradiol-17a Upregulates Pyruvate Kinase M2Expression to Coactivate Estrogen Receptor- $\alpha$ and tolntegrate Metabolic Reprogramming With theMitogenic Response in Endometrial Cells, J Clin Endocrinol Metab, October 2014, 99(10):3790-3799

24. Israelsen WJ, Vander Heiden MG. Pyruvate kinase: Function, regulation and role in cancer. Semin Cell Dev Biol. 2015 Jul;43:43-51. doi: 10.1016/j.semcdb.2015.08.004. Epub 2015 Aug 13.

25. Vander Heiden MG, Locasale JW, Swanson KD, et. al. Evidence for an alternative glycolytic pathway in rapidly proliferating cells. Science. 2010 Sep 17;329(5998):1492-9. doi: 10.1126/science.1188015.

26. Hsu MC, Hung WC. Pyruvate kinase M2 fuels multiple aspects of cancer cells: from cellular metabolism, transcriptional regulation to extracellular signaling. Mol Cancer. 2018 Feb 19;17(1):35. doi: 10.1186/s12943-018-0791-3.

27. Hardt PT, Mazurek S, Toepler M, et al. Faecal tumour M2 pyruvate kinase: a new, sensitive screening tool for colorectal cancer. $\mathrm{Br} J$ Cancer. 2004 Aug 31;91(5):980-4. doi: 10.1038/sj.bjc.6602033 
28. Ventrucci M, Cipolla A, Racchini C, Casadei R, Simoni P, Gullo L. Tumor M2-Pyruvate Kinase, a New Metabolic Marker for Pancreatic Cancer. Dig Dis Sci 2004, 49, 1149-1155. doi: 10.1023/B:DDAS.0000037803.32013.aa

29. Mazurek S, Drexler HC, Troppmair J, Eigenbrodt E, Rapp UR.Regulation of pyruvate kinase type M2 by A-Raf: a possible glyco-lytic stop or go mechanism.Anticancer Res. 2007;27:39633971.

30. Clower CV, Chatterjee D, Wang Z, Cantley LC, Vander HeidenMG, Krainer AR.The alternative splicing repressors hnRNP A1/A2and PTB influence pyruvate kinase isoform expression and cell me-tabolism.Proc Natl Acad Sci USA. 2010:2;107(5):1894-1899.

31. Moncada S, Higgs EA, Colombo SL.Fulfilling the metabolic re-quirements for cell proliferation.Biochem J. 2012;446:1-7.

32. DeBerardinis RJ, Thompson CB.Cellular metabolism and disease: what do metabolic outliers teach us?Cell. 2012;148:1132-1144

33. Ning $X$, Qi H, Li R, Li $Y$, Jin $Y$, McNutt MA, et al. Discovery of novel naphthoquinone derivatives as inhibitors of the tumor cell specific M2 isoform of pyruvate kinase. Eur $\mathrm{J}$ Med Chem. (2017) 138:343-52

34. Zhao X, Zhu Y, Hu J, Jiang L, Li L, Jia S, et al. Shikonin inhibits tumor growth in mice by suppressing pyruvate kinase M2-mediated aerobic glycolysis. Sci Rep. (2018) 8:14517

35. Ning X, Qi H, Li R, Jin Y,McNuttMA, Yin Y. Synthesis and antitumor activity of novel 2, 3didithiocarbamate substituted naphthoquinones as inhibitors of pyruvate kinase M2 isoform. $J$ Enzyme Inhib Med Chem. (2018) 33:126-9 


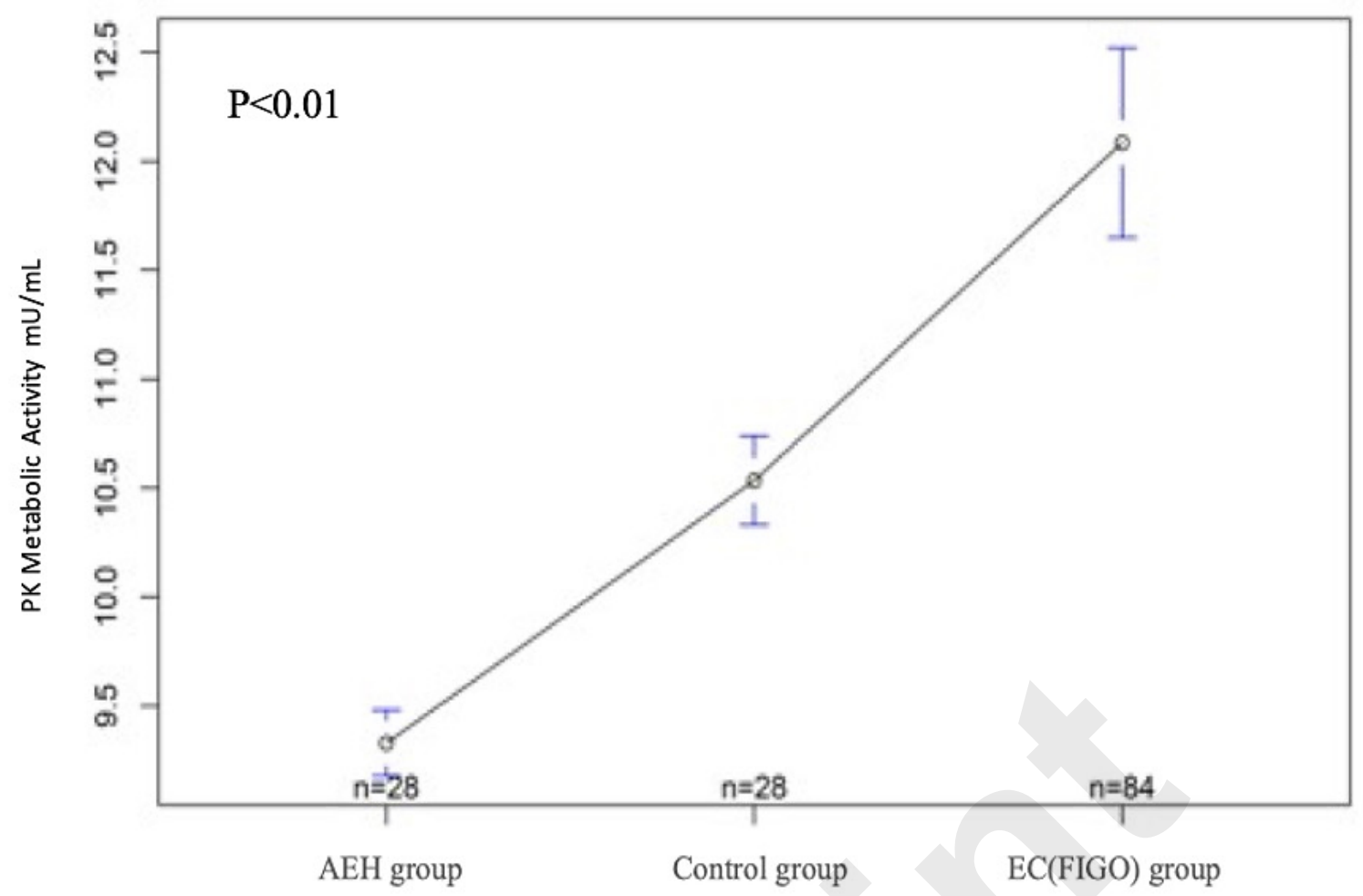

Figure 1. Peripheral blood M2 pyruvate kinase (PK) activity in patients with atypical endometrial hyperplasia $(\mathrm{AEH})$ or endometrial cancer $(\mathrm{EC})$ and healthy controls. Mean values $\pm S D$ are as presented. $P<0.01$ was observed between all pairs. 


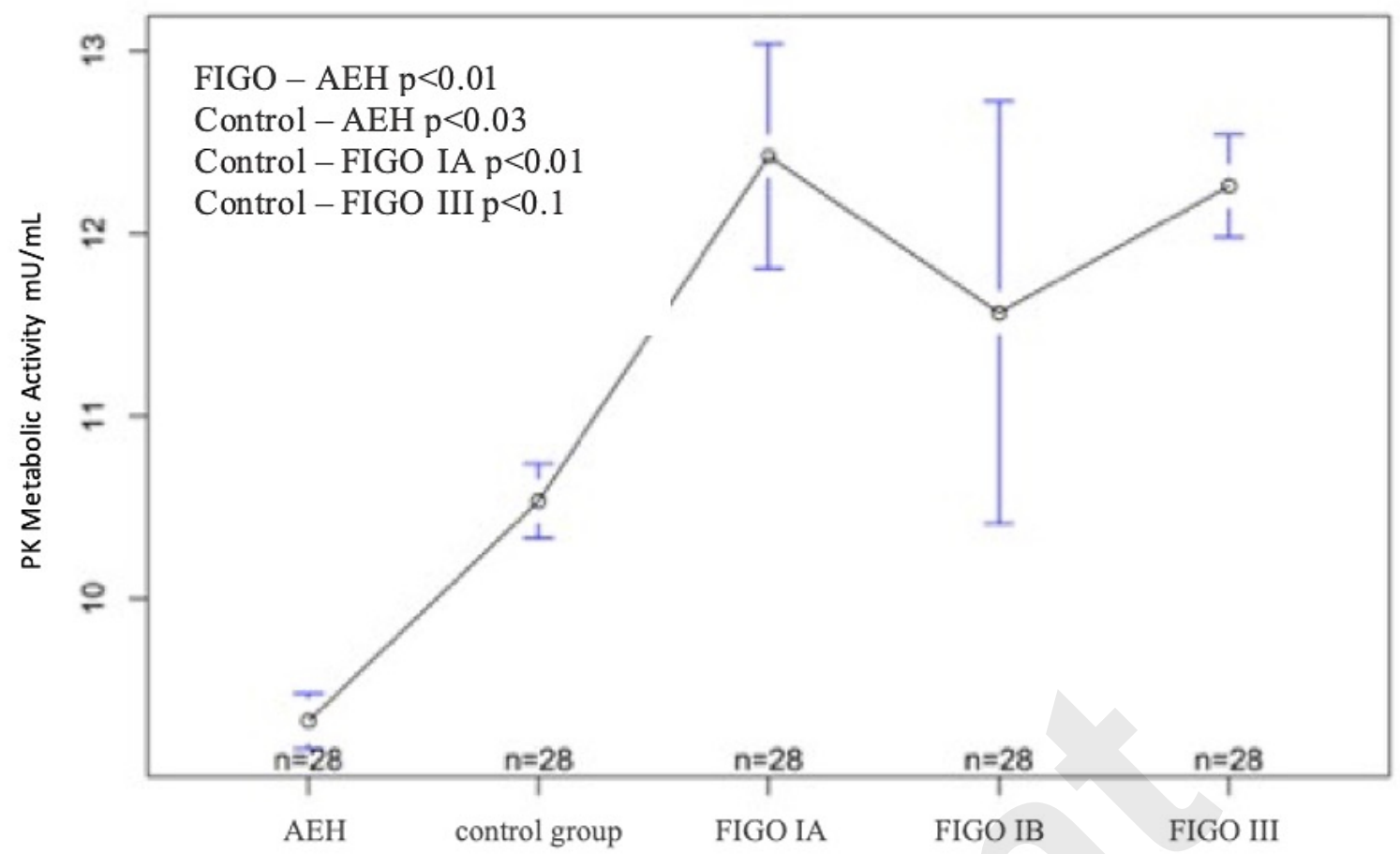

Figure 2. Peripheral blood M2-type pyruvate kinase (PK) activity in patients with atypical endometrial hyperplasia (AEH), healthy controls and endometrial cancer staged into FIGO $I A$, FIGO IB, FIGO > III, groups. Mean values $\pm S D$ are as presented. $P$ values were as follows: FIGO - AEH p <0.01, Control - AEH p $<0.03$, Control - FIGO IA $p<0.01$, Control FIGOIII $p<0.01$. 


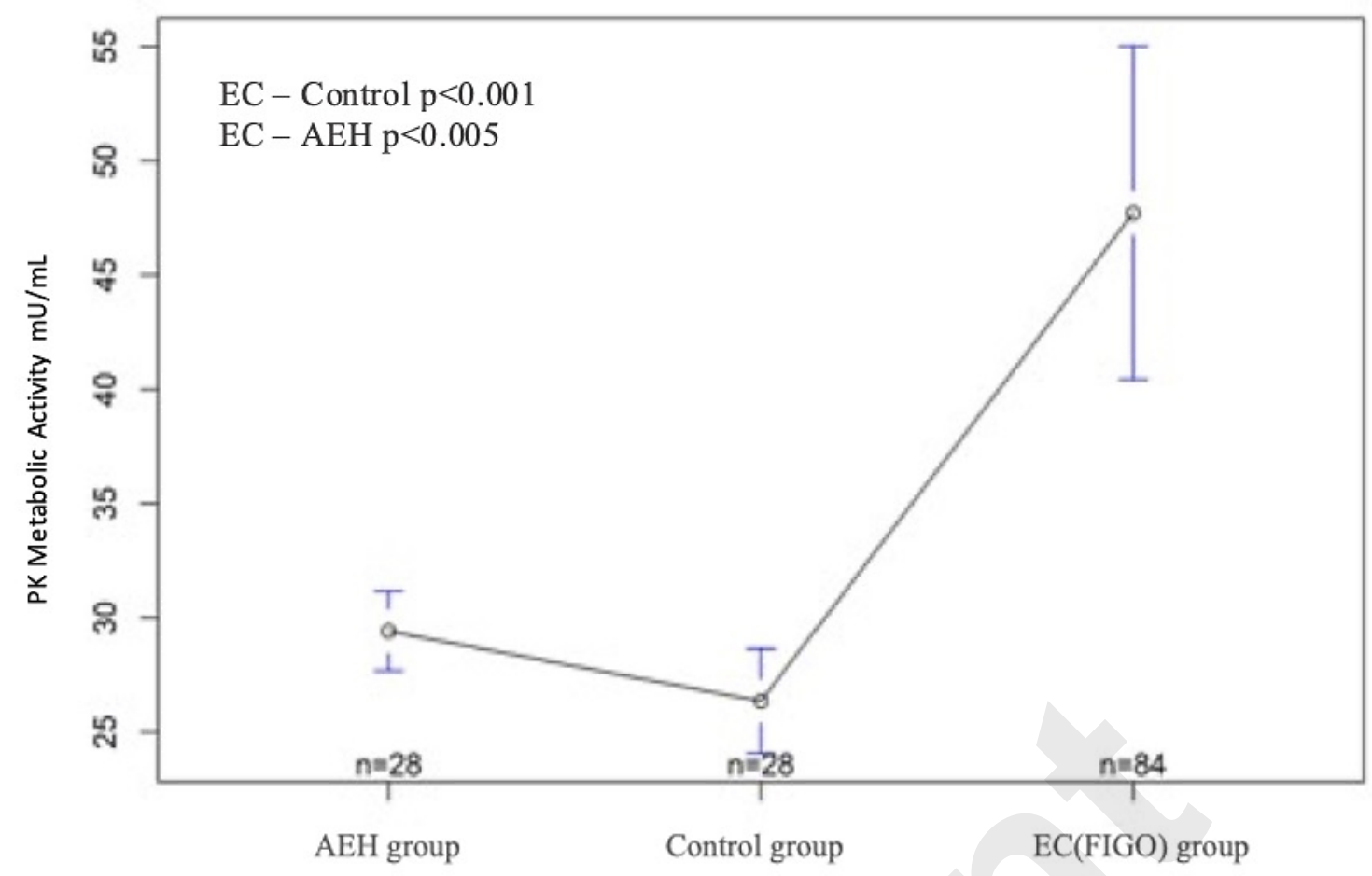

Figure 3. Uterine blood M2-type pyruvate kinase (PK) activity in patients with atypical endometrial hyperplasia (AEH) or endometrial cancer (EC) and healthy controls. Mean values $\pm S D$ are as presented. $P$ values were as follows: Endometrial cancer - control $p<0.001$, endometrial cancer $-\mathrm{AEH} p<0.005$. 


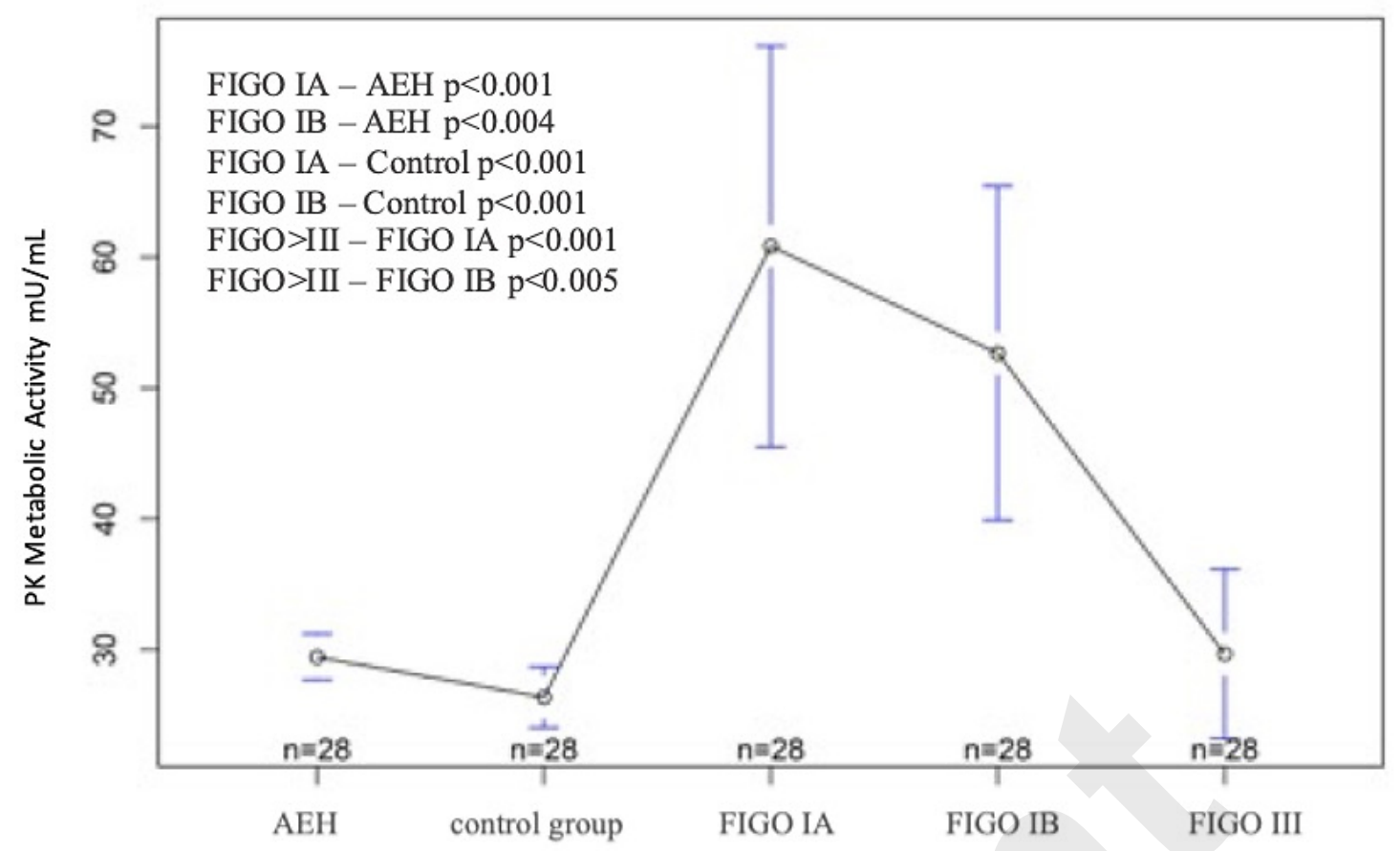

Figure 4. Uterine blood M2-type pyruvate kinase (PK) activity in patients with atypical endometrial hyperplasia $(\mathrm{AEH})$, healthy controls and endometrial cancer staged into FIGO $I A, F I G O I B, F I G O>I I I$, groups. Mean values $\pm S D$ are as presented. $P$ values were as follows: FIGO IA - AEH $p<0.001$, FIGO IB - AEH $p<0.004$, FIGO IA - control $p<0.001$, FIGO 1B - control $p<0.001$, FIGO $>$ III - FIGO IA $p<0.001$, FIGO $>$ III - FIGO IB $p<0.005$. 


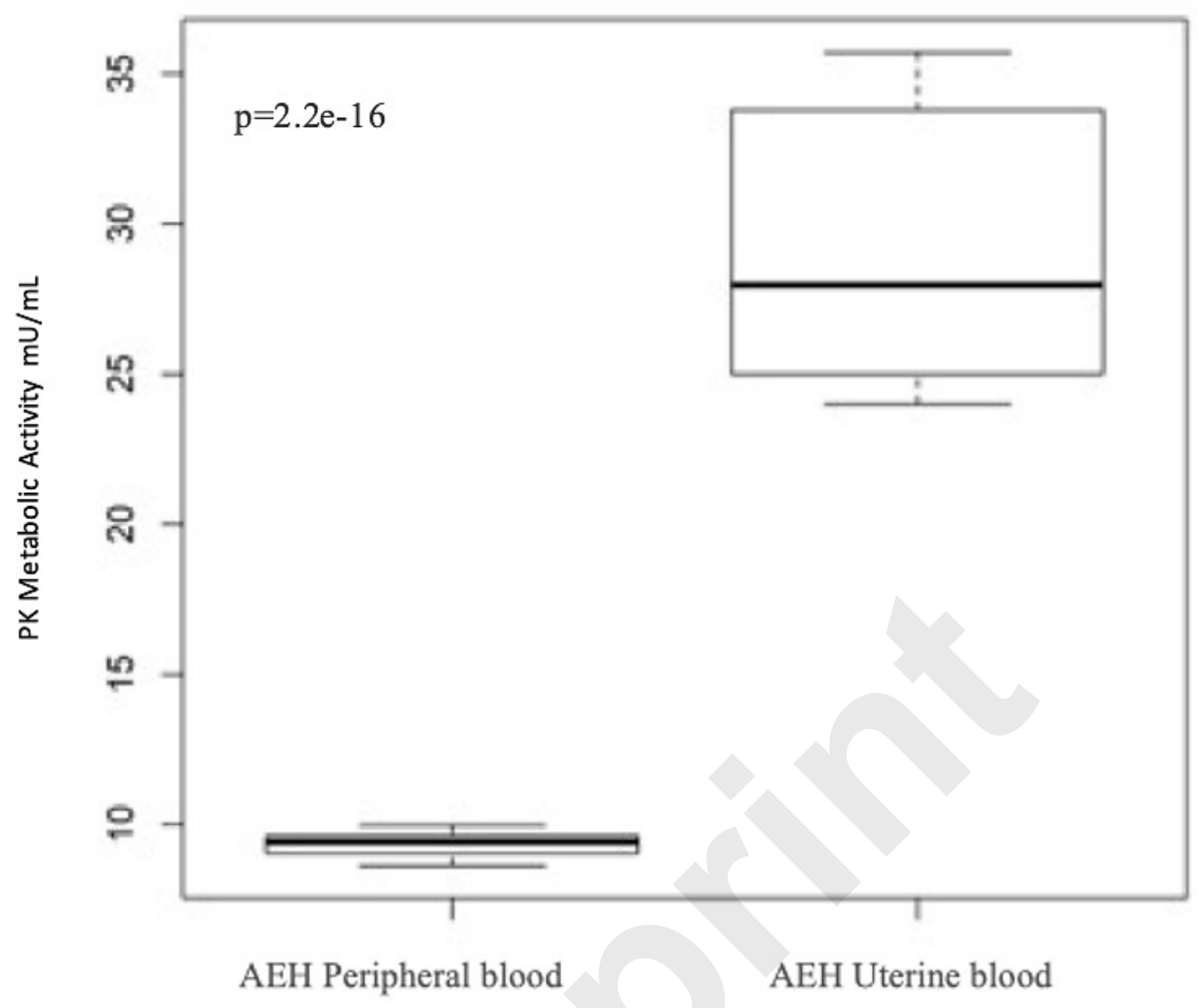

Figure 5. Boxplot with a range restriction to exclude major outliers for mean values of M2-type pyruvate kinase (PK) activity in peripheral and uterine blood of women with atypical endometrial hyperplasia (AEH). $p=2.2 e-16$. 


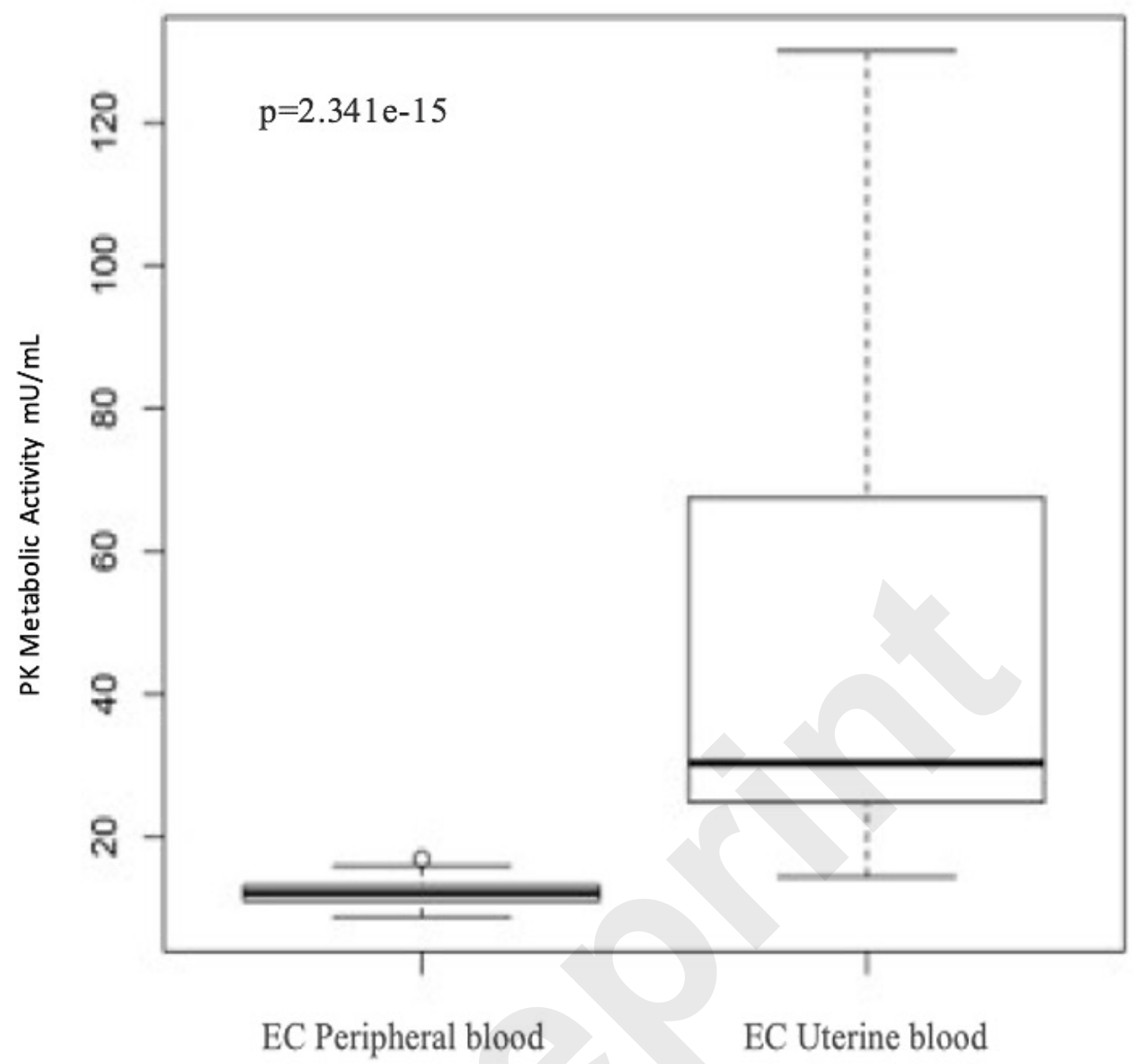

Figure 6. Boxplot with a range restriction to exclude major outliers for mean values of M2-type pyruvate kinase activity (PK) in peripheral and uterine blood of women with endometrial cancer (EC). $\mathrm{P}=2.341 \mathrm{e}-15$. 